\title{
Discours
}

Revue de linguistique, psycholinguistique et informatique. A journal of linguistics, psycholinguistics and computational linguistics

$15 \mid 2014$

Varia

\section{Matériels sur tout + gérondif}

\section{Oreste Floquet}

\section{OpenEdition}

\section{Journals}

\section{Édition électronique}

URL : http://journals.openedition.org/discours/8962

DOI : 10.4000/discours.8962

ISSN : 1963-1723

Éditeur :

Laboratoire LATTICE, Presses universitaires de Caen

\section{Référence électronique}

Oreste Floquet, « Matériels sur tout + gérondif », Discours [En ligne], 15 | 2014, mis en ligne le 19 décembre 2014, consulté le 03 mai 2019. URL : http://journals.openedition.org/discours/8962 ; DOI : $10.4000 /$ discours.8962

\section{(c) $(1)(9)$}

Discours est mis à disposition selon les termes de la licence Creative Commons Attribution - Pas d'Utilisation Commerciale - Pas de Modification 4.0 International. 

Revue de linguistique, psycholinguistique et informatique

\section{Matériels sur tout + gérondif}

Oreste Floquet

DSEAI

Sapienza - Université de Rome

Oreste Floquet, «Matériels sur tout + gérondif», Discours [En ligne], 15 | 2014, mis en ligne le 19 décembre 2014.

URL: http://discours.revues.org/8962

Titre du numéro: Varia

Coordination: Catherine Bolly et Benjamin Fagard 



\title{
Matériels sur tout + gérondif
}

\author{
Oreste Floquet \\ DSEAI \\ Sapienza - Université de Rome
}

Le but de cet article est d'apporter de nouvelles données sur la séquence «tout + gérondif» en français contemporain, en utilisant des analyses quantitatives (deux corpus oraux, un corpus écrit) et qualitatives (tests métalinguistiques de paraphrase et d'acceptabilité). D'un point de vue quantitatif, il semblerait émerger que tout + gérondif soit une forme très peu fréquente, généralement placée après le verbe recteur, et qu'on emploie de préférence avec des prédicats atéliques. D'un point de vue qualitatif, on peut constater que l'éventail de possibilités syntactiques et sémantiques de tout + gérondif est plus ample que celui qui a été proposé par les analyses introspectives précédentes et que les valeurs que les locuteurs choisissent suivent une certaine logique. Les données semblent montrer aussi qu'au-delà de l'opposition et de la simultanéité, tout + gérondif pourrait indiquer le cadre temporel, l'inclusion et la cause. Par ailleurs, cette tournure semble tolérer un sujet implicite différent du sujet du verbe support. Pour finir, puisque tout + gérondif fonctionne probablement comme un «converbe de phrase» (mettant donc en corrélation deux propositions plus ou moins autonomes), il ne peut pas indiquer la manière.

Mots clés: gérondif, approches quantitatives, compétence métalinguistique, linguistique de corpus, syntaxe et sémantique

The goal of this article is to provide new empirical data on "tout + gerund" in present day French by means of quantitative (two corpora of spoken French, one corpus taken from the French press) and qualitative analyses (two metalinguistic tests based on paraphrase and acceptability). From a statistical point of view, it seems that tout + gerund is not very frequent, it is often placed after the principal verb and it expresses atelic events. From a qualitative point of view, the syntactic and semantic possibilities of tout + gerund seem to be wider than those suggested by previous introspective analyses, and speakers seem to follow a certain logic on choosing such values. Beyond opposition and synchrony, our data show that tout + gerund can express temporal location, inclusion and cause. Moreover, it tolerates an implicit subject that may be different from the subject of the main verb. Finally, since it is probably a "phrasal converb" (as it correlates two more or less independent clauses) it cannot indicate manner.

Keywords: gerund, quantitative approach, metalinguistic awareness, corpus linguistics, syntax and semantics

\section{Introduction ${ }^{1}$}

Dans le cadre des études sur le gérondif en français contemporain, la tournure tout + gérondif est restée jusqu'ici peu explorée. Voulant reprendre le flambeau des recherches de Halmøy (1990: 195), qui termine son article pionnier par cette phrase:

1. Je tiens à remercier les deux experts anonymes dont les réactions précises et pertinentes m'ont beaucoup aidé à améliorer la version finale de cet article. Je reste bien entendu le seul responsable des imperfections subsistantes. 
$[\mathrm{U}] \mathrm{n}$ examen systématique des paraphrases possibles, du rôle de la place des syntagmes - Tout $G$ antéposé ou postposé au verbe recteur -, des types de texte, ou d'autres paramètres encore, pourra jeter une lumière différente sur la question ${ }^{2}$.

on se propose de fournir ici quelques nouvelles considérations quantitatives et qualitatives sur tout + gérondif, en donnant la priorité à l'examen des données brutes fournies par les corpus.

On abordera le problème de l'incidence statistique de tout + gérondif, de sa distribution syntaxique par rapport au verbe recteur ainsi que de la fréquence des lexèmes verbaux utilisés. Par ailleurs, on affrontera les problèmes syntaxiques et sémantiques de ce tour de manière non introspective. Puisque les rares commentaires des linguistes sont parfois contradictoires entre eux et fondés, pour l'essentiel, sur leurs jugements intuitifs, on proposera une analyse basée sur l'observation des données objectives. Il s'agira de présenter et d'interpréter les résultats d'une série de tests de paraphrase et d'acceptabilité dans le but de proposer une analyse faisant état de la compétence métalinguistique d'un certain nombre de locuteurs; cela nous évitera ainsi d'avancer des hypothèses qui ne peuvent être falsifiées puisqu'elles se fondent uniquement sur l'intuition du linguiste qui les émet.

\section{Analyse quantitative : quelques tendances syntaxiques, sémantiques et pragmatiques}

\subsection{Aspects statistiques généraux}

La présence du syntagme gérondif, quoique globalement assez modeste, varie considérablement si le corpus est oral ou bien écrit: dans le français parlé, nous avons une moyenne de 3,4 gérondifs tous les ıooo mots alors que, dans la presse, ce chiffre atteint $13, \mathrm{I}^{3}$. Toujours en restant sur un plan quantitatif, on relève que dans nos corpus le tour tout + gérondif est fortement minoritaire par rapport à la forme sans tout, dans une proportion quasi identique à l'oral et à l'écrit ${ }^{4}$ :

2. Dans la suite de cet article on va se référer pour l'essentiel à la bibliographie spécifique concernant tout + gérondif. Sur le gérondif simple (sans tout), on lira avec profit, entre autres, Arnavielle (2003 et 2010), Halmøy (2003) et Kleiber (2007).

3. Pour plus de précisions sur les attestations relativement modestes du gérondif simple dans la presse et à l'oral spontané, voir Floquet et al. (2012) et Escoubas-Benveniste et al. (2012). Ces données ont été récemment confirmées par l'étude menée par Escoubas-Benveniste (2013), qui est le troisième volet d'une analyse quantitative et qualitative effectuée sur les énoncés authentiques dans les deux modalités expressives (elle s'appuie sur un corpus de français écrit d'environ 6800000 mots et de français oral d'environ 3200000 mots).

4. On ne prend pas en considération les gérondifs grammaticalisés qui ont un tout autre statut; voir Halmøy (2003: 77-79), Floquet et al. (2012: 2145), Stosic (2012) et Vigier (2012). Le corpus écrit analysé ici se compose de l'ensemble des articles de L'Humanité de l'année 2010 (ci-après LH); il s'agit d'une partie du fonds d'archives numériques de l'université de Franche-Comté, le «Scriptorium numérique FANUM», dirigé par Jean-Marie Viprey (40 MB de texte équivalant à 6873329 occurrences). Le premier corpus oral analysé représente 529543 occurrences provenant de la base de données du projet international intitulé «Phonologie du français contemporain» (ci-après PFC), qui est l'un des corpus de français parlé les plus importants actuellement disponibles. Il est en ligne à l'adresse suivante: http://www.projet-pfc.net. 


\begin{tabular}{|c|c|c|c|c|c|c|}
\hline \multirow[b]{3}{*}{$\mathrm{N}$ tot gér } & \multicolumn{4}{|c|}{ Corpus oraux } & \multirow{2}{*}{\multicolumn{2}{|c|}{$\begin{array}{l}\text { Corpus écrit } \\
\text { LH }\end{array}$}} \\
\hline & \multicolumn{2}{|c|}{$\mathrm{PFC}$} & \multicolumn{2}{|c|}{ CFPP } & & \\
\hline & 182 & $100 \%$ & 170 & IOO \% & 9038 & $100 \%$ \\
\hline $\mathrm{N}$ tout gér & I3 & $7,2 \%$ & 9 & $5,2 \%$ & 738 & $8, \mathrm{I} \%$ \\
\hline N gér & 169 & $92,8 \%$ & I6I & $94,8 \%$ & 8300 & $91,9 \%$ \\
\hline
\end{tabular}

Tableau 1. Présence de tout + gérondif dans les trois corpus ${ }^{5}$

Comme on essaiera de le montrer par la suite, la marginalité du tour tout + gérondif est un aspect à prendre sérieusement en compte dans son analyse. Si on considère qu'une telle forme est peu fréquente, on comprend peut-être mieux son comportement très instable du point de vue syntaxique et surtout sémantique, qui est dû à son statut faible dans le système ${ }^{6}$.

\subsection{Aspects distributionnels et informatifs}

Comme le rappelle Halmøy, sur le plan syntaxique, tout + gérondif peut être antéposé à son support verbal (position frontale), postposé (position finale) ou intercalé entre le sujet et son verbe (position interne). Dans le premier cas, il s'interprète comme étant thématique, dans le deuxième comme rhématique; placé entre le sujet et le verbe, il serait indifféremment thématique ou rhématique en fonction du cotexte ${ }^{7}$. Contrairement aux remarques de Barra-Jover (1995:6) pour qui :

la structure traitée tend à l'ordre tout en..., Ph(rase) plutôt que l'inverse

nos données montrent que les positions initiales et internes sont rarissimes aussi bien à l'oral qu'à l'écrit et que le devant de la scène est monopolisé par tout + gérondif en position finale ${ }^{8}$ :

On s'est limité aux enquêtes menées sur le territoire métropolitain (Is départements répartis sur tout le territoire, pour un total de 280 dialogues analysés). Concernant le deuxième corpus oral, il s'agit du «Corpus de français parlé parisien» (ci-après CFPP), qui est composé d'un ensemble d'interviews auprès d'habitants de la région Île-de-France portant sur les quartiers de Paris et de la proche banlieue. Ce corpus est disponible à l'adresse suivante: http://www.cfpp2ooo.univ-paris3.fr. Il correspond à 36,6 heures d'enregistrement, soit 483 I52 occurrences.

5. $\mathrm{PFC}=$ Phonologie du français contemporain $; \mathrm{CFPP}=$ Corpus de français parlé parisien; $\mathrm{LH}=\mathrm{L}^{\prime} H u m a-$ nité, année 20I0. $\mathrm{N}$ tot gér = nombre total de gérondifs; $\mathrm{N}$ tout gér = nombre total de tout + gérondif; $\mathrm{N}$ gér $=$ nombre total de gérondifs sans tout.

6. Sur le rapport de la fréquence avec les zones plus ou moins fortes et stables de la grammaire, voir, entre autres, Giannini (2003: 127-134).

7. Voir Halmøy (2003: 127-I28).

8. Il faut rappeler que la position finale était canonique en ancien français, tout + gérondif occupant toujours la position postverbale. Ce n'est qu'après que les formes en -ant se voient dotées d'une fonction thématique en position frontale. Au vu de la situation contemporaine, il serait intéressant de mener une étude diachronique plus approfondie afin de comprendre si on a affaire à un changement syntaxique limitant les possibilités distributionnelles de tout + gérondif, comme au Moyen Âge. Sur cet aspect syntaxique, voir Combettes (2003) et Halmøy (2003: 43-44). Sur la diachronie de ces formes, dans une optique plus générale, voir Arnavielle (1997), Skårup (2006) et Zanola (1999). 


\begin{tabular}{|l|l|l|l|}
\hline & \multicolumn{2}{|l|}{ Corpus oraux } & Corpus écrit \\
\cline { 2 - 4 } & PFC & CFPP & LH \\
\hline Tout $G+$ SV (frontal) & 2 & 2 & 58 \\
\hline S + Tout $G+$ V (interne) & - & - & $3 \mathrm{I}$ \\
\hline SV + ToutG (final) & II & 7 & 649 \\
\hline
\end{tabular}

Tableau 2. Distribution de tout + gérondif par rapport au verbe support (nombre d'occurrences)

Concernant cette position finale non marquée, l'analyse des occurrences de PFC semble montrer que le tour tout + gérondif final fait partie intégrante du rhème du paragraphe oral, la position la plus saillante d'un point de vue informatif (Morel et Danon-Boileau, 1998). Ses propriétés prosodiques (la variation de Fo et l'absence de pause) et syntaxiques (structure $<$ Pro $+\mathrm{V}>+\mathrm{X}$ ) le distinguent assez bien des incises, des post-rhèmes et de tout élément peu intégré au noyau de l'énonciation ${ }^{9}$. En ce qui concerne l'écrit, ce type d'analyse s'avère encore plus difficile. Si on prend comme critère la ponctuation, même si celle-ci n'est jamais un calque parfait de la prosodie, on voit que seulement $47 \%$ des tours tout + gérondif finaux sont séparés de leur support verbal par une virgule ${ }^{10}$; il nous semble donc que même dans ce corpus, tout + gérondif est la plupart du temps lié au verbe support, occupant ainsi une position tendanciellement rhématique plutôt que celle d'un simple ajout faiblement informatif.

\subsection{Aspects lexicaux ${ }^{11}$}

D'après Halmøy (2003: 132), sur le plan de l'aspect lexical, à la différence de ce qui se passe pour le gérondif simple, le tour tout + gérondif est l'apanage surtout des verbes d'état dont, pour l'essentiel, être (+ adjectif, participe passé, ou SN) ou bien avoir (+ SN ou participe passé) ${ }^{12}$. Pourtant avoir n'est pas l'un des lexèmes les plus fréquents, puisque dans les trois corpus, son incidence est égale à zéro ou tout à fait marginale (contrairement à être qui est au premier rang aussi bien dans CFPP que dans LH) :

9. Plus précisément, dans les exemples extraits de PFC, on repère deux contours intonatifs qui signalent, selon Morel et Danon-Boileau (1998), que le verbe support et tout + gérondif forment un continuum ("coalescence sémantique») : soit il y a variation de Fo sur le SV - p. ex. 44ajs2_GG, la deuxième continue ses études pour passer une thèse ( $\mathrm{FO}_{\mathrm{O}}$ ) tout en donnant des cours à la fac-, soit on constate une double montée - p. ex. 44ajs2_GG, et ensuite j’ai passé mes diplômes ( $\mathrm{Fo}_{\mathrm{O}}$ ) tout en travaillant (Fo+). Sur ces aspects prosodiques, voir Morel et Danon-Boileau (1998: 50-53).

10. Sur la tendance générale de l'écrit à placer l'information la plus saillante à la frontière droite de la phrase et sur le fonctionnement, peu stable, de la virgule, voir Lombardi Vallauri (2002: 95-IO4); pour le cas du français, voir, entre autres, Béguelin (2012).

11. Sur les aspects théoriques qui concernent l'utilisation des corpus en sémantique, voir Condamines (2005).

12. Puisque cela ne changerait pas les statistiques, on ne va pas aborder ici la question du participe présent composé et de sa dénomination, pour laquelle on peut voir Herslund (2000). Pour quelques détails sur l'aspect lexical du gérondif sans tout, voir Floquet et al. (2012: 2153, n. I6). 


\begin{tabular}{|l|l|l|l|}
\hline \multirow{2}{*}{} & \multicolumn{2}{|l|}{ Corpus oraux } & Corpus écrit \\
\cline { 2 - 4 } & $\mathrm{PFC}$ & $\mathrm{CFPP}$ & $\mathrm{LH}$ \\
\hline $\mathrm{N}$ avoir/N total & $\mathrm{O} / \mathrm{I} 3$ & $\mathrm{O} / 9$ & $3 / 738$ \\
\hline Rang du lexème & - & - & $\mathrm{I} 6$ \\
\hline N être/N total & $\mathrm{I} / \mathrm{I} 3$ & $6 / 9$ & $27 / 738$ \\
\hline Rang du lexème & 2 & $\mathrm{I}$ & $\mathrm{I}$ \\
\hline
\end{tabular}

Tableau 3. Densité de être et avoir

À côté des verbes statiques, on repère une grande quantité de verbes exprimant des procès ou des actions, plutôt que des éventualités (Chierchia, 1997: 361-370). Dans le tableau 4, on transcrit les principaux lexèmes verbaux par ordre décroissant en signalant entre parenthèses le nombre effectif de mots-formes:

\begin{tabular}{|c|c|c|c|}
\hline & Corpus oraux & & Corpus écrit \\
\hline & PFC & CFPP & $\mathrm{LH}$ \\
\hline Rang I & $\begin{array}{l}\text { enseigner, travailler } \\
\left(2 / \mathrm{I}_{3}\right)\end{array}$ & $\begin{array}{l}\text { être } \\
(6 / 9)\end{array}$ & $\begin{array}{l}\text { être } \\
(30 / 738)\end{array}$ \\
\hline Rang 2 & $\begin{array}{l}\text { chercher, dire, donner, } \\
\text { etre, garder, babiter, } \\
\text { laisser, rester, descendre } \\
\left(\mathrm{I} / \mathrm{I}_{3}\right)\end{array}$ & $\begin{array}{l}\text { dire, habiter, passer } \\
(\mathrm{I} / 9)\end{array}$ & $\begin{array}{l}\text { faire } \\
(27 / 738)\end{array}$ \\
\hline $\operatorname{Rang}_{3}$ & - & - & $\begin{array}{l}\text { rester } \\
(23 / 738)\end{array}$ \\
\hline Rang 4 & - & - & $\begin{array}{l}\text { préciser } \\
(\mathrm{1} 7 / 738)\end{array}$ \\
\hline Rang 5 & - & - & $\begin{array}{l}\text { préserver, appeler } \\
(\mathrm{I} 4 / 738)\end{array}$ \\
\hline Rang 6 & - & - & $\begin{array}{l}\text { continuer, reconnaître } \\
\left(\mathrm{I}_{3} / 738\right)\end{array}$ \\
\hline Rang 7 & - & - & $\begin{array}{l}\text { affirmer } \\
(\mathrm{1} 2 / 738)\end{array}$ \\
\hline Rang 8 & - & - & $\begin{array}{l}\text { donner } \\
(\text { II/738) }\end{array}$ \\
\hline Rang 9 & - & - & $\begin{array}{l}\text { maintenir, garder, } \\
\text { refuser } \\
\text { (Io/738) }\end{array}$ \\
\hline Rang IO & - & - & $\begin{array}{l}\text { savoir } \\
(9 / 738)\end{array}$ \\
\hline
\end{tabular}

Tableau 4. Densité des lexèmes verbaux les plus fréquents 
$\mathrm{Si}$ on regroupe les verbes d'état et les verbes exprimant un procès non ponctuel, il semble émerger une deuxième tendance: tout + gérondif s'emploie de préférence avec des prédicats atéliques.

\section{Sur la sémantique de tout + gérondif}

La question de la sémantique de tout + gérondif - l'un des aspects les plus controversés de cette construction - a donné lieu à des analyses différentes, que l'on va brièvement esquisser ${ }^{13}$. La position radicale consiste à exclure toute interprétation non concessive. C'est, par exemple, ce qu'on retrouve dans la grammaire de Riegel et al. (2009: 592):

La préposition en peut être renforcée par l'adverbe tout, en particulier quand le gérondif exprime une opposition: Tout en étant dévot, on n'en est pas moins homme.

Pour Barra-Jover (1995:7) aussi, l'opposition est le seul trait qui soit intrinsèque aux formes avec tout:

Le français offre toute une série de segments qui, d'une part sont composés de l'élément tout et, d'autre part, transmettent un sens se rapprochant de la concessivité (ou adversativité) ${ }^{14}$.

Plus nuancée est la position à deux variables (opposition vs concomitance) comme celle de Franckel (1989) qui distingue l'apport invariable de tout dans la structure tout + adjectif/nom prédicatif/gérondif/adverbe $+P$, des effets contextuels découlant des deux sémantismes verbaux, celui du gérondif et du verbe support, qui peuvent donner lieu à des effets plus ou moins concessifs. Puisque le rôle de tout est de marquer une homogénéisation aspectuelle dans la relation gérondif-verbe, il s'ensuit que (Franckel, 1989:340):

la valeur sans pour autant cesser de n'apparaît qu'à condition que ce fonctionnement opère sur deux procès dont la nature sémantique tend à exclure la concomitance ${ }^{15}$.

Cela signifie qu'il y a deux effets possibles (simultanéité et contraste) qui sont sensibles au contexte et que, par voie de conséquence, l'opposition n'est pas un trait invariant de tout mais plus simplement l'interprétation la plus courante (Franckel, 1989: 340):

Cette valeur se neutralise dans un exemple comme Tout en me promenant, je repensais aux belles journées d'autrefois, mais le fonctionnement de tout demeure

13. Conscient des ambiguïtés de la notion d'«opposition», on va tout de même continuer de l'utiliser comme synonyme des notions de «concessif» et d'«adversatif».

14. Dans la même veine, voir aussi Kindt (1999: II5) pour qui tout indique que le procès exprimé par tout + gérondif ne peut pas être compatible, du point de vue sémantique, avec le procès exprimé par le verbe défini.

15. Halmøy aussi considère que, pour Franckel, l'opposition est la fonction sémantique la plus fondamentale du tour tout + gérondif, alors que la neutralisation ne serait qu'un cas particulier. 
identique, même si l'altérité introduite par la seconde proposition n'est plus aussi nettement adversative $[. .$.$] .$

Plus détaillé, le système de Halmøy se structure à nouveau sur deux macrofonctions. Partant de la constatation que tout opère un blocage de l'interprétation instrumentale, de cause et de manière (tout disjonctif), par rapport à la forme simple du syntagme gérondif, elle voit dans tout - et il semblerait que ce soit là sa valeur fondamentale - une réfutation de divers types de topoï. Ce tout polyphonique est soit une trace syntaxique de l'énonciation, soit un marqueur d'une concomitance qui ne serait toutefois que purement fortuite.

Pour Grevisse aussi, il y a deux options dépendant du contexte verbal, même si chez lui c'est plutôt la valeur concessive qui semble être marquée, par rapport à la valeur de concomitance qui demeure la plus prototypique:

En faisant précéder en de l'adverbe tout, on souligne la simultanéité, le contexte suggérant souvent une nuance d'opposition ${ }^{16}$.

Dans le même sillage, nous trouvons également l'analyse de Rihs (2013: 19I), pour qui tout + gérondif impose d'abord la simultanéité, la concession n'étant qu’un «complément optionnel».

Un système à trois variables est celui de Gettrup (cité par Halmøy), le seul qui intègre aux deux fonctions (temporelle et oppositive) la possibilité que tout puisse être aussi sémantiquement vide (Halmøy, I990: 188):

Il semble que tout apparaisse parfois dans des emplois où aucune des explications précédentes ne convienne et où il ne sert qu'à donner plus de poids au syntagme. Gettrup qualifie ce dernier emploi de «stylistique».

Gettrup admet donc qu'il existe des cas où l'apport de tout est pratiquement nul, ce qui rend identiques les formes du gérondif simple et de tout + gérondif ${ }^{17}$.

Reprenant les considérations critiques de Moline (20II) sur la possibilité d'attribuer au gérondif simple des valeurs sous-jacentes sans le recours à des connaissances pragmatiques, on va maintenant s'interroger sur la possibilité concrète de déterminer de manière stable et univoque le sémantisme de tout + gérondif. Comme le rappelle Caron (2008: 16):

Les processus par lesquels l'esprit humain traite le langage échappent, pour la plus grande part, à l'introspection. On ne peut les aborder que par des méthodes indirectes ${ }^{18}$.

16. Voir M. Grevisse, Le bon usage, Louvain-la-Neuve, De Boeck-Duculot, § 926 c, version électronique, http://www.lebonusage.com. Dans la même veine, voir aussi Bat-Zeev Shyldkrot (1995).

17. Grevisse signale aussi le contraire, à savoir des cas où la nuance concessive est possible sans qu'il y ait le tout.

18. En faveur d'une démarche expérimentale en sémantique, voir aussi Le Ny (1987). 
C'est pourquoi, afin de court-circuiter la méthode courante chez les linguistes qui consiste à se prendre soi-même comme sujet interrogé, on a conçu deux expériences inspirées des travaux sur la compétence métalinguistique ${ }^{19}$.

\subsection{Une étude sur le processus de compréhension: la paraphrase}

Un premier test est centré sur la paraphrase et vise à explorer les jugements d'acceptabilité et de similitude d'un nombre consistant de locuteurs natifs du français (Fuchs, 1982: 28):

Les risques de la méthode introspective en matière de constitution de données paraphrastiques sont pourtant importants. Le linguiste ne se comporte en effet pas comme un sujet parlant quelconque: ce constat s'impose. Tantôt il établira des relations paraphrastiques là où un locuteur, dans l'exercice concret du langage, ne le ferait pas [...]. Tantôt, au contraire, il récusera des relations de paraphrase qui «passeraient» très bien pour des locuteurs en situation [...].

Pour ce qui est de la structure du questionnaire, la consigne a été la suivante:

Cochez la ou les paraphrases qui vous semblent les plus correctes; s'il n'y en a pas, vous pouvez proposer la vôtre (au point «h. Autre») ${ }^{20}$.

Les informateurs devaient donc choisir parmi les huit réponses possibles (forcedchoice experiment) ${ }^{21}$. En voici un exemple:

19. Comme le rappelle Gombert (I990 et 1996), le terme de métalinguistique varie si l'approche est linguistique ou psycholinguistique. Dans le droit fil de Pinto - dont le travail mené depuis une vingtaine d'années représente le soubassement théorique et méthodologique incontournable qui est à l'origine de la conception de ces expériences (voir, entre autres, Pinto [1999]; Pinto et Iliceto [2007]) -, on considère la métalinguistique comme étant une activité de réflexion et de contrôle sur le langage. Concernant les tests, ils ont été soumis à 37 informateurs de langue maternelle française. Nous sommes tout à fait conscient que d'un point de vue purement quantitatif, le nombre de personnes interrogées est encore insuffisant pour arriver à des conclusions irréfutables. Il s'agit d'un premier échantillon exploratoire qu'il va falloir de toute évidence élargir (le deuxième volet de cette enquête fait partie des projets financés par le programme de recherche "Sapienza 2013»). Voici tout de même quelques renseignements sur ces 37 informateurs qui ont participé aux deux expériences (l'âge varie de 18 à 30 ans) :

(a) Provenance (35, Paris; 2, Nantes);

(b) Sexe (26 femmes, 11 hommes);

(c) Niveau d'études ( 19 , niveau bac à bac $+3 ; 18$, niveau bac +4 jusqu'au doctorat $)$.

La soumission du questionnaire a été effectuée à Paris par Francesca De Felice entre avril et mai 2013.

20. Cette expérience reprend en partie l'épreuve de compréhension mise au point par Pinto qui sert à mesurer la compétence métasémantique des rapports qualitatifs, temporels, morphologiques et spatiotemporels.

21. Sur la différence entre des expériences de paraphrase de type free-response (où le choix est totalement libre et imprévisible) ou bien de type forced-choice (où les informateurs sont contraints par des options préalablement établies), voir Gleitman et Gleitman (I970: I05-I48 et 233-234). Notre expérience s'inspire aussi du test de paraphrase de Solarino (I996), encore que celui-ci ne soit pas basé sur la comparaison des jugements de plusieurs locuteurs mais uniquement sur les intuitions de l'auteur. 
I) Tout en jouant du piano, il regarde la télé.

\begin{tabular}{|l|}
\hline a. Bien qu'il joue du piano, il regarde la télé. \\
\hline b. Il regarde la télé, donc il joue du piano. \\
\hline c. Il regarde la télé, encore qu'il joue du piano. \\
\hline d. Il regarde la télé, mais il joue du piano. \\
\hline e. Il regarde la télé, pendant qu'il joue du piano. \\
\hline f. Puisqu'il joue du piano, il regarde la télé. \\
\hline g. Tandis qu'il joue du piano, il regarde la télé. \\
\hline h. Autre................................................................. \\
\hline
\end{tabular}

Comme on peut le constater, les marqueurs peuvent appartenir - ou pas - à la même catégorie. Au vu de la littérature sur tout + gérondif, certains d'entre eux sont attendus, d'autres moins:

- réponse a, concessive logique (bien que);

- réponse $b$, consécutive (donc);

- réponse c, concessive rectificative (encore que) ${ }^{22}$;

- réponse d, adversative (mais);

- réponse e, concomitance (pendant que);

- réponse $\mathrm{f}$, causale marquant la présupposition (puisque) ${ }^{23}$;

- réponse g, repère temporel (tandis que ${ }^{24}$.

Quant aux inputs dont il fallait fournir une ou plusieurs équivalences, la plupart ont été choisis à partir d'exemples qui ont déjà été commentés par les linguistes qui se sont penchés sur la sémantique de ce tour:
A) Tout en jouant du piano, il regarde la télé;
B) Il peut donc rester Français, tout en ayant l'Algérie pour terre;
C) Tout en le considérant l'un des anarchistes les plus dangereux, la police allemande a perdu sa trace;
D) Tout en vidant son verre, il éclate de rire;
E) Le président s'attelle à la réconciliation avec le Maroc et la France, tout en se rapprochant de Washington;

22. Sur la différence entre une concession logique et une concession rectificative ainsi que sur leur distribution, voir Morel (1996).

23. Sur la présupposition dans puisque, voir Nazarenko (2000).

24. D'après Gettrup (1977:224) : «En principe, un pendant que/tandis que antéposé est presque toujours perçu comme un repère temporel». 
F) Tout en continuant à lire comme si de rien n'était, il le surveille du coin de l'œil;

G) Ils finissent par accepter l'idée d'un État palestinien, tout en refusant à ce dernier les attributs de la souveraineté;

$\mathrm{H})$ Il rougit, tout en la regardant;

I) La maison se trouve tout en descendant à gauche derrière le camping municipal;

L) Elle joue du piano, tout en s'appliquant.

Cela a au moins deux avantages manifestes: (I) on a pu travailler sur des interprétations préexistantes qui ne nous appartiennent pas forcément, évitant ainsi de retomber dans les pièges de l'introspection (ce qui aurait été le cas si on était parti de nos propres interprétations); (2) on a pu mesurer le degré de ressemblance des intuitions des linguistes par rapport à celles des non-spécialistes, créant ainsi un mini-corpus de jugements métalinguistiques exploitable de manière objective. Il faut ajouter aussi que, par souci d'équilibrage des données, on a parfois interverti l'ordre des constituants afin qu'il y ait un nombre presque égal de tout + gérondif en position frontale et finale, et que toutes les phrases ont été transformées à la troisième personne du singulier.

Il ne faut pas non plus oublier que ce type de questionnement présente un certain nombre de limites qui ont été largement mises en évidence par la littérature scientifique (Caron, 2008: 18-19; Fuchs, 1982: 32-38). C'est pourquoi la nature essentiellement analytique de notre démarche, visant à interpréter les réactions à des séquences données, a été assouplie en introduisant la possibilité de produire une paraphrase autre que celles proposées (réponse «h. Autre»); ce qui laisse au sujet davantage de liberté de choix. Une autre limite évidente de notre démarche est le fait que le questionnement est explicite et direct puisque les paraphrases ont été présentées comme étant toutes possibles, ce qui réduit au minimum la possibilité de signaler l'agrammaticalité et invite subrepticement l'informateur à trouver toujours une interprétation plausible. Enfin, pour remédier au manque de contexte explicite - ce qui aurait rendu la collecte des données plus longue et plus laborieuse -, on a donné la possibilité d'opter pour plusieurs paraphrases possibles en même temps, selon les différentes situations d'énonciation envisageables par les locuteurs eux-mêmes ${ }^{25}$.

25. Sur l'importance du contexte dans le paraphrasage (avec des exemples français), voir Fuchs (I994). Pour une théorie du langage axée sur la paraphrase, on lira avec profit Milićević (2007). Quant au gérondif simple (sans tout), il faut rappeler les réserves de Halmøy (2003: 89-9I), pour qui la substitution par une subordonnée circonstancielle n'est pas un critère sûr. Le problème se pose tout autrement pour tout + gérondif, puisque les interprétations se polarisent uniquement autour de la concomitance ou de la concession, ce qui nous autorise, ce nous semble, à opter pour une démarche visant à explorer, même si c'est de façon approchée, les jugements métalinguistiques de nos informateurs par le biais du test de paraphrase. 


\subsection{Analyses quantitatives et qualitatives}

On propose de classer les réponses en trois catégories selon que leur distribution parmi les informateurs est plus ou moins homogène. À des cas où n'émerge qu'une seule valeur (D, H et I), s'opposent des situations où l'on constate une hétérogénéité extrême (L), en passant par des tests où les réponses se polarisent autour de deux ou trois valeurs (A, B, C, E, F et $\mathrm{G})$.

\subsubsection{Distribution homogène forte: repère temporel et causalité}

On va d'abord étudier deux batteries de tests où le pourcentage de réponses concernant une seule valeur, en l'occurrence temporelle, atteint plus de $70 \%$ des interviewés. Pour cette typologie de réponses, on propose l'étiquette de «distribution forte», contrairement aux deux autres cas de I dont la distribution, bien qu'homogène, est néanmoins plus faible puisque l'accord n'atteint que $40 \%$ des informateurs.

D'après Halmøy (2003: 130-13I), le tour tout + gérondif ne peut ni être un repère temporel - c'est-à-dire qu'il ne peut pas marquer un intervalle de temps ample à l'intérieur duquel une action se produit alors qu'il peut indiquer une relation de simultanéité fortuite que l'on peut gloser moyennant une coordination -, ni indiquer l'antériorité logique, ce qui empêche toute coloration causale ${ }^{26}$.

Dans le cas de l'input $\mathrm{D}$, le procès exprimé par syntagme gérondif ne peut pas être coïncident au procès du verbe recteur dont l'aspect est ponctuel. Il faut plutôt le considérer comme un cadre à l'intérieur duquel se déroule l'action d'éclater de rire. Les réponses sont très nettes et montrent une majorité écrasante de choix pour pendant que et tandis que qui, d'après Gettrup (1977: 224), peuvent indiquer aussi bien la concomitance que le cadrage temporel. Les interprétations concessive et adversative, quoique possibles, sont, en revanche, presque absentes. Voici les résultats pour D:

\begin{tabular}{|l|l|l|}
\hline \multicolumn{2}{|l|}{} & Total \\
N \\
\hline a & Bien que & 4 \\
\hline b & Donc & - \\
\hline c & Encore que & - \\
\hline d & Mais & I \\
\hline e & Pendant que & 27 \\
\hline f & Puisque & - \\
\hline g & Tandis que & 25 \\
\hline h & Autre & I \\
\hline
\end{tabular}

26. Du même avis, Moline (20II : IOI) rappelle toutefois, avec Gettrup, que «malgré l'existence de critères permettant de distinguer les Vger de localisation temporelle des Vger de concomitance, il ne s'agit pas de deux classes totalement distinctes $[\ldots] »$. 
Bien que ces données méritent d'être validées par d'autres expériences plus ciblées, on ne peut pas exclure que l'action du verbe recteur puisse être incorporée dans celle qui est exprimée par le tour tout + gérondif ${ }^{27}$. Un informateur, d'ailleurs, tout en choisissant l'option tandis que, a ressenti le besoin aussi d'ajouter à la ligne «h. Autre» le gérondif simple en vidant, ce qui semble confirmer qu'il a interprété le rapport des deux procès comme étant inclusif.

Les résultats pour $\mathrm{H}$ montrent la même possibilité:

\begin{tabular}{|c|c|c|}
\hline & & $\begin{array}{l}\text { Total } \\
\mathrm{N}\end{array}$ \\
\hline $\mathrm{a}$ & Bien que & 3 \\
\hline b & Donc & - \\
\hline c & Encore que & I \\
\hline d & Mais & I \\
\hline e & Pendant que & 27 \\
\hline f & Puisque & 8 \\
\hline g & Tandis que & 23 \\
\hline h & Autre & - \\
\hline
\end{tabular}

L'input a été repris à quelques détails près de Halmøy (2003: 130), qui considère la présence de tout comme peu acceptable ou carrément agrammaticale. Et pourtant deux considérations semblent s'imposer. Premièrement, aucun des informateurs n'a signalé au point "h. Autre» une quelconque insatisfaction par rapport à la grammaticalité de la phrase; deuxièmement, la présence tout à fait exceptionnelle de huit réponses avec puisque (ce qui correspond à $22 \%$ des informateurs) pourrait signaler que les deux procès maintiennent une certaine autonomie puisqu'on ne peut pas exclure a priori une coloration causale, encore que faible, au sens de Moeschler (2003:25), à côté de la valeur d'arrière-plan (à l'intérieur duquel l'action de rougir se déroule) ${ }^{28}$.

\subsubsection{Distribution homogène faible: la coréférence du sujet implicite}

Dernier cas où les jugements des informateurs sont assez nets, bien que quantitativement moins importants par rapport à $\mathrm{D}$ et $\mathrm{H}$ : les réponses concernant I. On sait qu'à l'oral il existe des gérondifs non canoniques pouvant prendre la place d'un participe présent (Floquet et al., 2012: 2148 sq.). Pour I, l'anomalie est d'ordre

27. Voir infra les résultats du test d'acceptabilité.

28. On a repéré, dans les exemples extraits de PFC, une autre occurrence où l'interprétation causale semble plausible: donc tout en cherchant à se loger dans les environs eub ils ont décidé de s'installer définitivement dans cet endroit-là parce que c'était joli (Izaası_GG). Sur les rapports entre cause, gérondif et participe, voir Rihs (2009). 
syntaxique et concerne plutôt la non-coréférence du sujet implicite du gérondif et du sujet du verbe recteur, ce qui semblerait devoir être interdit puisque, selon Halmøy (2003: 13I):

il s'agit toujours de deux actions ou procès menés de front par un même agent.

29. ESLO est une initiative entreprise en 1969 consistant à collecter, à des fins didactiques, des document sonores illustrant le français parlé. Ce corpus est en ligne à l'adresse suivante: http://eslo.huma-num.fr.

30. Il semble que, compte tenu de la sémantique de l'énoncé, le locuteur, plus ou moins consciemment, évite de recourir à toute manifestation syntaxique des agents du procès à travers un pronom sujet, fût-il indéfini ("même si on se connaît... on a une rivalité...»). En taisant ce lien de référence syntaxique, l'énonciateur opère une sorte de neutralisation des acteurs impliqués dans un paradoxe plutôt délicat - sujets de «se connaître» et d'une "rivalité... cinglante... méchante». Cette neutralisation prudente se poursuit syntaxiquement par l'usage de la forme impersonnelle «il y a une rivalité». Nous devons ces éléments d'analyse à Escoubas-Benveniste. 
Une considération encore sur ces exemples. On pourrait objecter que notre argumentation est très peu concluante du fait qu'elle se base uniquement sur trois exemples, encore que diatopiquement éloignés. Sans vouloir abandonner la prudence qui est de rigueur quand on travaille sur des données brutes, il faut néanmoins rappeler que la forme tout + gérondif semble être globalement assez rare à l'oral, ce qui a été récemment confirmé par Escoubas-Benveniste (2013) ${ }^{31}$.

Passons maintenant au tableau des réponses des informateurs:

\begin{tabular}{|l|l|l|}
\hline \multicolumn{2}{|l|}{} & $\begin{array}{l}\text { Total } \\
\text { N }\end{array}$ \\
\hline a & Bien que & 2 \\
\hline b & Donc & 5 \\
\hline c & Encore que & 3 \\
\hline d & Mais & 3 \\
\hline e & Pendant que & I5 \\
\hline f & Puisque & 3 \\
\hline g & Tandis que & I3 \\
\hline h & Autre & 6 \\
\hline
\end{tabular}

À la différence de ce qui se passe pour $\mathrm{D}$ et $\mathrm{H}$, où le choix pour les réponses «e. Pendant que» et «g. Tandis que» atteint plus de $70 \%$ des informateurs, ici la concentration est inférieure, quoique significative ( $40 \%$ de réponses pour pendant que et $35 \%$ pour tandis que). Chose remarquable, le nombre de réponses «h. Autre» augmente. Il s'agit de six informateurs qui, par ailleurs, n'ont opté pour aucun des choix proposés. Cela paraît confirmer que la non-coréférence des sujets demeure une structure syntaxique possible quoique très marquée ${ }^{32}$.

\subsubsection{Distribution polarisée: rapports implicatifs}

On va passer maintenant aux six tests dont la concentration des choix est partagée entre deux ou trois valeurs. Au vu des réponses des informateurs, la thèse suivant laquelle tout donnerait toujours une coloration concessive au gérondif semble devoir être remise en question. En dépit des résultats de son enquête, Barra-Jover (1995: 32, n. 5) considère que l'énoncé $A$ n'a pas d'autre coloration que concessive:

Lorsque nous avons invité certains informateurs à nous donner des exemples non concessifs, ils ont proposé des énoncés qui indiquent l'étonnement face à l'exécution

31. L'exploitation des corpus en syntaxe est d'ailleurs un sujet très débattu (Oliviéri, 2oıo) donnant lieu à un rejet complet par certains courants de la linguistique contemporaine (Longobardi, 2003), qui pointent du doigt le fait que les données sont souvent quantitativement très réduites par rapport à celles dont on dispose en phonologie où ce genre d'enquête ne pose pas les mêmes problèmes.

32. Sur le problème du sujet implicite, voir Reichler-Béguelin (1995). 
simultanée de deux actions. P. ex. : [i] Tout en jouant du piano, il regarde la télé. En fait, nous y repérons aisément le sens concessif.

Or, l'analyse des réponses de nos informateurs montre, en revanche, que des deux options concessives, «c. Encore que» n'est jamais choisie alors que «a. Bien que», qui est loin d'être majoritaire, ne représente qu'un peu plus de la moitié des choix concernant «e. Pendant que»:

\begin{tabular}{|l|l|l|}
\hline \multicolumn{2}{|l|}{} & $\begin{array}{l}\text { Total } \\
\text { N }\end{array}$ \\
\hline a & Bien que & I3 \\
\hline b & Donc & - \\
\hline c & Encore que & - \\
\hline d & Mais & 7 \\
\hline e & Pendant que & 25 \\
\hline f & Puisque & - \\
\hline g & Tandis que & 22 \\
\hline h & Autre & I \\
\hline
\end{tabular}

L'option concessive n'est pas la plus attestée: c'est plutôt la temporalité, qu'elle soit exprimée par «e. Pendant que» ou par «g. Tandis que», qui a été davantage choisie comme paraphrase plausible. On retrouve la même situation avec les énoncés $E$ et $\mathrm{F}$. Dans une étude sur la traduction des constructions détachées du français à l'espagnol - notamment celle de tout + gérondif - dont le but est d'évaluer les choix de traducteurs pour comprendre les stratégies cognitives qui leur sont sous-jacentes, Andújar Moreno (20I0) présente $\mathrm{E}$ comme un exemple de lecture uniquement temporelle, ce qui semble n'être confirmé qu'en partie par nos données, lesquelles montrent en revanche que l'interprétation concessive est tout de même bien représentée car elle est acceptable pour $35 \%$ des informateurs (Andújar Moreno, 20I0: 48):

\begin{tabular}{|l|l|l|}
\hline \multicolumn{2}{|l|}{} & $\begin{array}{l}\text { Total } \\
\text { N }\end{array}$ \\
\hline a & Bien que & I3 \\
\hline b & Donc & I \\
\hline c & Encore que & 4 \\
\hline d & Mais & 8 \\
\hline e & Pendant que & 28 \\
\hline f & Puisque & 2 \\
\hline g & Tandis que & I9 \\
\hline h & Autre & - \\
\hline
\end{tabular}


Dans $\mathrm{F}$ on retrouve la même situation:

\begin{tabular}{|l|l|l|}
\hline \multicolumn{2}{|l|}{} & Total \\
N \\
\hline a & Bien que & I9 \\
\hline b & Donc & I \\
\hline c & Encore que & 4 \\
\hline d & Mais & I6 \\
\hline e & Pendant que & 24 \\
\hline f & Puisque & - \\
\hline g & Tandis que & 20 \\
\hline h & Autre & - \\
\hline
\end{tabular}

Majoritaire dans l'ensemble des dix enquêtes, la valeur temporelle, qu'elle soit exprimée par pendant que ou bien par tandis que, est en revanche quantitativement plus modeste dans les tests B, C et G. Dans B, le nombre d'informateurs ayant choisi les options concessive et adversative reste faible (38\%), encore que supérieur au nombre de ceux qui ont choisi la valeur temporelle pendant que (qui représente toutefois presque la moitié des choix de bien que et mais) ${ }^{33}$ :

\begin{tabular}{|l|l|l|}
\hline \multicolumn{2}{|l|}{} & Total \\
N \\
\hline a & Bien que & I4 \\
\hline b & Donc & - \\
\hline c & Encore que & 9 \\
\hline d & Mais & I4 \\
\hline e & Pendant que & 6 \\
\hline f & Puisque & - \\
\hline g & Tandis que & 5 \\
\hline h & Autre & 3 \\
\hline
\end{tabular}

L'énoncé C est considéré par Gettrup (1977), Halmøy (2003) et Cuniţă (20II) comme étant un exemple flagrant de cause contrariée du moment qu'il

implique une acceptation générale selon laquelle le côté dangereux de l'homme demanderait la plus grande vigilance, ce qui n'est apparemment pas le cas ${ }^{34}$.

33. L'exemple B est tiré de Halmøy (I990: I83).

34. Gettrup, cité par Halmøy (I990: I88). Voir aussi Cuniţă (20II : 66). 
Et pourtant, les deux réponses majoritaires semblent s'équilibrer avec un léger avantage de bien que sur tandis que:

\begin{tabular}{|l|l|l|}
\hline \multicolumn{2}{|l|}{} & $\begin{array}{l}\text { Total } \\
\text { N }\end{array}$ \\
\hline a & Bien que & 20 \\
\hline b & Donc & - \\
\hline c & Encore que & 5 \\
\hline d & Mais & I2 \\
\hline e & Pendant que & I \\
\hline $\mathrm{f}$ & Puisque & - \\
\hline $\mathrm{g}$ & Tandis que & $\mathrm{I} 7$ \\
\hline $\mathrm{h}$ & Autre & 2 \\
\hline
\end{tabular}

La chute de pendant que au profit de tandis que - qui peut ajouter une nuance d'opposition même si, dans la paraphrase proposée, sa position est frontale et donc susceptible de marquer le cadre temporel (Tandis qu'elle le considère l'un des anarchistes les plus dangereux, la police allemande a perdu sa trace) -, semble indiquer que, pour les informateurs, la valeur oppositive est la plus naturelle. Les réponses pour l'énoncé $G$ aussi sont tendanciellement concessives ou adversatives :

\begin{tabular}{|l|l|l|}
\hline \multicolumn{2}{|l|}{} & $\begin{array}{l}\text { Total } \\
\text { N }\end{array}$ \\
\hline a & Bien que & 25 \\
\hline b & Donc & - \\
\hline c & Encore que & 20 \\
\hline d & Mais & 24 \\
\hline e & Pendant que & 6 \\
\hline f & Puisque & I \\
\hline g & Tandis que & I7 \\
\hline h & Autre & - \\
\hline
\end{tabular}

Andújar Moreno (2010: 50) présente cette phrase comme un exemple de lecture exclusivement concessive, déclenchée par les antonymes accepter et refuser, mais comme on peut le constater, l'option temporelle n'est pas écartée pour autant par les informateurs.

Enfin, il importe de souligner que contrairement à $\mathrm{D}, \mathrm{H}$ et $\mathrm{I}$, où l'écart entre la valeur temporelle (pendant que et tandis que) et les valeurs concessive (bien que et encore que) et adversative (mais) est très important, dans ces trois derniers tests 
aucun des choix attendus ne prime réellement sur les autres. L'objectif de cette recherche n'étant pas la mise en évidence des conditions sémantiques déclenchant la valeur oppositive, on se borne juste à constater qu'une telle interprétation, quand bien même elle serait majoritaire, n'exclut jamais une lecture temporelle, alors que le contraire n'est pas vrai. Il nous semble que ce résultat remet en cause les approches, comme celle de Franckel (1989), où la relation oppositive est considérée comme étant prototypique par rapport à la valeur temporelle, et va plutôt dans la direction de Rihs (2013: I9I) pour qui «la concession procède de la simultanéité» ${ }^{35}$.

\subsubsection{Distribution aléatoire: tout + gérondif et l'expression de la manière}

On va terminer par le seul test qui nous a donné des réponses sans aucune concentration significative des outputs; il s'agit de l'énoncé L: Elle joue du piano, tout en s'appliquant. Conformément aux intuitions de Halmøy (2003: 133), qui reprend sur ce point Bonnard, il semble aussi émerger de nos données que tout + gérondif ne peut pas exprimer la manière dont l'action se déroule:

\begin{tabular}{|c|c|c|}
\hline & & $\begin{array}{l}\text { Total } \\
\mathrm{N}\end{array}$ \\
\hline $\mathrm{a}$ & Bien que & I \\
\hline b & Donc & 8 \\
\hline c & Encore que & 2 \\
\hline $\mathrm{d}$ & Mais & 9 \\
\hline e & Pendant que & 8 \\
\hline $\mathrm{f}$ & Puisque & I \\
\hline g & Tandis que & 9 \\
\hline h & Autre & 7 \\
\hline
\end{tabular}

Comme on peut le constater, la plus grande dispersion des réponses par rapport aux autres tests, de même que le nombre de réponses «h. Autre» (dont trois explicitent on ne peut plus clairement que c'est la forme sans le tout qui serait la

35. Cela ne signifie pas non plus que la valeur temporelle est obligatoirement sous-jacente aux autres. On peut citer sur ce point la position de Moline (2OII : 92), qui affirme à propos du gérondif simple que: «[...] la temporalité ne correspond pas à une valeur première du gérondif, mais au contraire à une relation assignée par défaut, quand nos connaissances du monde nous interdisent d'inférer d'autres relations». Il est important de rappeler que d'un point de vue diachronique, la valeur adversative est toujours, du moins dans les langues romanes, le fruit du développement d'une valeur temporelle indiquant la continuité; voir Giacalone Ramat et Mauri (20I2) et aussi Bat-Zeev Shyldkrot (1995: 86) qui reprend certaines considérations de Traugott concernant le fait que la simultanéité finit, dans de nombreuses langues, par exprimer une opposition (voir, p. ex., Traugott [1989]). Un exemple éclairant de transformation diachronique d'une valeur temporelle à une valeur concessive est celui de cependant; voir Marchello-Nizia (2007). 
plus correcte), semblent indiquer que, même si les locuteurs interviewés ont ressenti l'énoncé comme étant très peu acceptable, ils ont tout de même essayé de répondre en reformulant souvent l'énoncé de départ ${ }^{36}$.

\subsection{Le test d'acceptabilité}

Dans notre deuxième expérience, on a adapté l'épreuve d'acceptabilité de Pinto (1999) et Pinto et Iliceto (2007), où on vérifie la capacité des sujets à repérer, corriger et justifier les incongruités présentes dans un texte préétabli. Dans cette épreuve, l'item est constitué par l'anomalie elle-même, incorporée dans le texte, que le sujet doit reconnaitre. La consigne que l'on a fournie aux 37 informateurs était la suivante:

Dans le texte suivant vous trouverez un certain nombre d'incongruités. Indiquez-les et corrigez-les. Ensuite, justifiez chaque correction.

Le texte de départ était le suivant:

Des squelettes ont été déterrés lors de travaux dans la City, à Londres, et les experts pensent qu'il pourrait s'agir d'un immense charnier abritant des victimes de la "peste noire», au Moyen Âge. Les ossements de treize adultes, soigneusement alignés, ont été découverts à 2,5 mètres de profondeur dans le quartier financier de la capitale britannique, lors d'excavations menées en vue de la construction d'une ligne ferroviaire.

Des tests doivent encore être réalisés, mais les archéologues pensent que beaucoup de victimes de la peste noire ont été enterrées à cet endroit, comme l'ont rapporté des documents du $\mathrm{XVI}^{\mathrm{e}}$ siècle suggérant qu'il pourrait y avoir jusqu'à cinquante mille cadavres. «C'est une découverte très importante», a souligné Jay Carver, qui dirige l'équipe d'archéologues qui travaille sur ce projet ferroviaire.

«Nous allons mener des tests sur les squelettes dans les mois à venir pour établir les causes de leur mort, pour voir s'il s'agit de victimes de la peste du XIV e siècle ou de résidents d'une époque ultérieure, pour déterminer leur âge et tenter de trouver des indices afin de savoir qui ils étaient. Mais à ce stade, tout semble indiquer qu'il s'agit de ce cimetière établi en urgence au XIV siècle», a-t-il ajouté. Les scientifiques espèrent aussi en apprendre plus sur cette épidémie de peste noire et sur le virus qui en est responsable.

Quand les examens seront terminés, les ossements seront à nouveau enterrés, au même endroit ou dans un autre cimetière. Les archéologues espèrent aussi trouver des objets datant de la période romaine pendant leurs fouilles. Une fois que celles-ci seront achevées, des travaux de forage seront menés à cet endroit dans le cadre du projet «Crossrail».

Cette nouvelle ligne doit traverser la capitale britannique d'est en ouest, reliant notamment la City à l'aéroport de Heathrow, pour désengorger le réseau de transports en commun londonien.

Près de $\mathrm{I}, 5$ million de Britanniques ont péri de la peste noire au XIV ${ }^{\mathrm{e}}$ siècle, soit près d'un tiers de la population, une maladie qui a tué au total 25 millions d'Européens.

36. Pour une interprétation de ce type de blocage, voir les "Considérations finales». 
Le texte modifié que l'on a proposé contenait des incongruités d'ordre phonographique (p. ex. soigneusement > soignesement), morphosyntaxique (p. ex. qui ils $>$ qu'ils) et sémantique (p. ex. causes $>$ prétextes). On a mis en petites capitales celles qui nous intéressent davantage pour cette expérience et en italique les autres:

Des squelettes ont été déterrées lors de travaux dans la City, à Londres, et les experts pensent qu'il pourrait s'agir d'un immense charnier abritant des victimes de la «peste noire», au Moyen Âge. Les ossements des treize adultes, soignesement alignés, ont été découverts à 2,5 mètres de profondeur dans le quartier financier de la capitale britannique, TOUT EN MENANT DES EXCAVATIONS en vue de la construction d'une ligne ferroviaire.

«C'est une découverte très importante», a souligné Jay Carver, dirigeant l'équipe d'archéologues qui travaille sur ce projet ferroviaire. «Nous allons mener des tests sur les squelettes dans les mois à venir en établissant ainsi les prétextes de leur mort, TOUT EN VOYANT s'il s'agit de victimes de la peste du XIVe siècle ou de résidents d'une époque ultérieure, pour déterminer leur âge et tenter de trouver des indices afin de savoir qu'ils étaient. Mais à ce stade, tout semble indiquer qu'il s'agit de ce fameux cimetière établi en urgence au XIV e siècle EN ÉTANT PROHIBÉ par les règlements», a-t-il ajouté.

Quand les examens seront terminés, les ossements seront à nouveau enterrés, au même endroit ou dans un autre cimetière. Près de 1,5 million de Britanniques ont péri de la peste noire au XIV ${ }^{e}$ siècle, soit près d'un tiers de la population, car il s'agit d'une maladie qui a tué au total 25 millions d'Européens.

Dans le premier des trois segments textuels que l'on va étudier plus en détail, une subordonnée temporelle introduite par lors de est transformée en une construction avec tout + gérondif:

\begin{tabular}{|c|c|}
\hline Texte de départ & Texte modifié \\
\hline $\begin{array}{l}\text { Les ossements de treize adultes, soigneuse- } \\
\text { ment alignés, ont été découverts à } 2,5 \text { mètres } \\
\text { de profondeur dans le quartier financier de } \\
\text { la capitale britannique, lors d'excavations } \\
\text { menées en vue de la construction d'une ligne } \\
\text { ferroviaire. }\end{array}$ & $\begin{array}{l}\text { Les ossements des treize adultes, soignesement } \\
\text { alignés, ont été découverts à } 2,5 \text { mètres de } \\
\text { profondeur dans le quartier financier de la } \\
\text { capitale britannique, TOUT EN MENANT DES } \\
\text { EXCAVATIONS en vue de la construction d'une } \\
\text { ligne ferroviaire. }\end{array}$ \\
\hline
\end{tabular}

Puisque seulement douze informateurs ont détecté cette incongruité et l'ont corrigée moyennant, pour l'essentiel, des paraphrases à valeur de cadrage (alors que, lors, pendant que, à l'occasion de) par rapport à l'action du verbe défini (ont été découverts), on est en droit, ce nous semble, de déduire que pour la majorité, la forme est acceptable en tant que repère temporel; cela confirme les résultats du test de paraphrase. Il est d'ailleurs important de signaler que parmi les douze informateurs qui ont apporté des corrections, il y en a un qui a juste placé tout en menant en tête de phrase - position prototypique des cadres temporels -, en justifiant son choix 
par un souci de clarté ${ }^{37}$. Cela dit, il faut aussi ajouter que le taux de corrections varie en fonction du type d'anomalie et du type d'informateur, ce qui empêche de généraliser excessivement ces résultats, du moins à ce stade de la recherche ${ }^{38}$.

Les résultats du deuxième segment étudié sont eux aussi très instructifs.

\begin{tabular}{|c|c|}
\hline Texte de départ & Texte modifié \\
\hline $\begin{array}{l}\text { Nous allons mener des tests sur les squelettes } \\
\text { dans les mois à venir pour établir les causes de } \\
\text { leur mort, pour voir s'il s'agit de victimes de } \\
\text { la peste du XIV siècle ou de résidents d'une } \\
\text { époque ultérieure, pour déterminer leur âge } \\
\text { et tenter de trouver des indices afin de savoir } \\
\text { qui ils étaient. }\end{array}$ & $\begin{array}{l}\text { Nous allons mener des tests sur les squelettes } \\
\text { dans les mois à venir en établissant ainsi les } \\
\text { prétextes de leur mort, TOUT EN vOYANT s'il } \\
\text { s'agit de victimes de la peste du XIV siècle } \\
\text { ou de résidents d'une époque ultérieure, pour } \\
\text { déterminer leur âge et tenter de trouver des } \\
\text { indices afin de savoir qu'ils étaient. }\end{array}$ \\
\hline
\end{tabular}

Seuls huit informateurs ont éprouvé le besoin de modifier le texte, interprétant correctement qu'à la source il devait y avoir une construction finale (pour vérifier, pour voir, afin de déterminer). Cela dit, trois d'entre eux ont repéré une anomalie lexicale, plutôt que syntaxique, puisqu'ils ont proposé tout en cherchant, tout en analysant et tout en vérifiant. Peut-on dire, pour ces derniers ainsi que pour ceux qui ne sont pas intervenus du tout, que la nuance de cause finale n'est pas complètement exclue? Rien ne le prouve de manière catégorique car on peut toujours faire intervenir la concomitance (mener des tests et voir s'il s'agit des victimes de la peste). D’autres enquêtes, plus amples et plus ciblées, devront éclaircir ce point. Reste que dans les deux cas, les actions ne fusionnent pas dans un seul événement mais font partie de deux situations distinctes (ce qui marque une différence par rapport au fonctionnement du gérondif simple) ${ }^{39}$.

On va maintenant passer au troisième segment.

\begin{tabular}{|c|c|}
\hline Texte de départ & Texte modifié \\
\hline $\begin{array}{l}\text { "Mais à ce stade, tout semble indiquer qu'il } \\
\text { s'agit de ce cimetière établi en urgence au } \\
\text { XIV siècle», a-t-il ajouté. }\end{array}$ & $\begin{array}{l}\text { «Mais à ce stade, tout semble indiquer } \\
\text { qu'il s'agit de ce fameux cimetière établi en } \\
\text { urgence au XIV siècle EN ÉTANT PROHIBÉ par } \\
\text { les règlements», a-t-il ajouté. }\end{array}$ \\
\hline
\end{tabular}

Deux aspects nous semblent remarquables. D'une part, seulement deux personnes ont corrigé en ajoutant tout, alors que des douze qui ont modifié cette partie du texte, la plupart l'ont fait en indiquant des marqueurs de concession; d'autre part,

37. Un cas plus ou moins comparable avec les trois anomalies que l’on va étudier plus en détail est celui de qui ils > qu'ils, où le nombre d'interventions atteint I9, à savoir presque la moitié des informateurs.

38. On sait d'ailleurs que généralement les monolingues sont moins performants dans ce type de tâche que les bilingues (Pinto, 20II).

39. Voir infra les «Considérations finales». 
la majorité des informateurs n'est pas intervenue. Ce type de résultats semblerait appuyer une intuition que l'on retrouve chez Grevisse et qui est rarement utilisée par les spécialistes, à savoir que la nuance oppositive n'est pas une marque exclusive de la construction tout + gérondif car on la repère dans le gérondif simple aussi ${ }^{40}$.

\section{Considérations finales}

Le but de cet article était d'apporter de nouvelles données quantitatives et qualitatives sur tout + gérondif en français contemporain, en poursuivant le travail séminal de Halmøy (1990: I8I) pour qui, à juste titre:

la question des emplois du gérondif précédé de tout reste peu étudiée et continue à poser des problèmes.

D’après nos premiers résultats provisoires il semble que : a) la position non marquée de tout + gérondif est post-verbale, à l'intérieur de la zone rhématique; b) l'éventail de possibilités syntaxiques et sémantiques de tout + gérondif est plus ample que celui qui a été proposé par les analyses introspectives précédentes (Franckel, 1989; Barra-Jover, 1995; Halmøy, 2003); c) la sémantique minimale de cette structure ne contient pas la dissonance et la disjonction, qui, en revanche, sont dérivables par inférence à partir du contexte ${ }^{41}$. Une telle plasticité n'est probablement pas sans rapport avec la rareté statistique et le statut marginal dans le système, qui ont pour résultat que ce tour soit plus malléable par rapport à d'autres structures de la langue.

Néanmoins, un certain nombre de questions importantes restent ouvertes. Puisque cela déborde quelque peu du cadre méthodologique de notre enquête exploratoire, on se borne juste à signaler quelques pistes de recherches possibles qui mériteraient des vérifications empiriques.

Plusieurs indices semblent indiquer que le point de rattachement de tout + gérondif pourrait être la phrase et non le verbe régissant, ou du moins une position intermédiaire entre le syntagme verbal et la phrase. Face à la négation, par exemple, les comportements syntaxiques de tout + gérondif et du gérondif simple, du moins en position post-verbale, semblent différents ${ }^{42}$. Dans [I'] et [2'], la négation du verbe défini n'a pas d'effet sur tout + gérondif:

[I] il parle tout en le regardant

[I] il ne parle pas tout en le regardant

40. M. Grevisse, Le bon usage, § 926 c, R2, version électronique, http://www.lebonusage.com: «la nuance d'opposition est possible sans tout: J'ai reconnu un Parisien à ce qu'il avait l'air anglais, et qu'EN AFFECTANT la raideur, il laissait voir une aisance parfaite et une vivacité toute particulière (FRANCE, Lys rouge, XXII)». Voir aussi Halmøy (2003: 134) et Rihs (2013: 190).

41. Dans le droit fil de Zanola (1998), on préfère partir plutôt de l'idée qu'il s'agit d'une forme implicite qui cache, en amalgame, un prédicat plus un connecteur qui dépend du contexte.

42. La position frontale, pré-verbale, mériterait probablement un discours à part. 
[2] il est devenu acteur tout en poursuivant son activité de musicien

[2'] il n'est pas devenu acteur tout en poursuivant son activité de musicien

alors qu'elle en a dans [3'] et [4'], où elle s'étend au gérondif simple :

[3] il évite la récession en baissant les impôts

[3'] il n'évite pas la récession en baissant les impôts

[4] il parle en élevant sa voix

[4'] il ne parle pas en élevant sa voix

La phrase [3'] signifie que le fait de baisser les impôts ne permet pas d'éviter la récession; la phrase [4'] signifie qu'il parle, mais sans élever sa voix.

Une deuxième différence concerne la difficulté bien connue de tout + gérondif à entrer dans une construction clivée ${ }^{43}$ :

\section{[5] c'est en forgeant qu'on devient forgeron \\ [5'] ?'ं'est tout en forgeant qu'on devient forgeron}

Là aussi on voit bien que le lien syntaxique avec le verbe n'est pas le même et que, dans le cas de tout + gérondif, il est moins étroit. Reprenant une distinction qui a été appliquée aux formes indéfinies d'autres langues romanes, notamment l'italien, on pourrait explorer l'hypothèse que, dans le premier cas, on a un «converbe de prédicat (ou propre)» représentant une modification circonstancielle à l'intérieur d'une phrase simple, et, dans le deuxième cas, un «converbe de phrase (ou coordonné)» mettant en corrélation deux propositions plus ou moins autonomes ${ }^{44}$. On sait d'ailleurs que d'un point de vue typologique, les converbes de phrase peuvent fonctionner aussi bien comme des propositions subordonnées que comme des propositions coordonnées ${ }^{45}$ et que les phénomènes de focalisation, comme dans [5], sont cantonnés pour l'essentiel aux phrases subordonnées ${ }^{46}$. On pourrait donc proposer, non sans beaucoup de prudence, que dans sa structure profonde tout + gérondif se situe à mi-chemin entre la subordination et la coordination, ce que depuis Van Valin (1984) on appelle la «co-subordination», et met toujours en place deux événements autonomes qui sont

43. Voir Halmøy (2003: I28) et Cuniţă (2011 : 66).

44. Suivant Nedjalkov (1995: 97), un «converbe» est une forme verbale qui dépend d'un autre verbe sans faire partie de ses actants. Pour une critique de la validité de cette catégorie linguistique, voir Zúñiga (1998). Sur les spécificités du converbe de phrase et de prédicat en italien, voir Lonzi (200I) et Mengozzi (I998). Pour une analyse contrastive du comportement de ces deux formes en italien et en français, voir Ramat et Da Milano (20II) et Floquet (20II).

45. Voir Nedjalkov (1995: 98-99) qui rappelle aussi qu'il ne s'agit pas de catégories rigides et étanches.

46. Voir Haspelmath (1995: 15). 
syntaxiquement subordonnés ${ }^{47}$. Dans le gérondif simple, en revanche, on aurait plutôt affaire à deux actions corrélées qui peuvent faire partie du même événement ${ }^{48}$. Si cela était vrai, on comprendrait peut-être mieux pourquoi ce tour est apte à exprimer aussi bien la concomitance que l'opposition et l'arrière-plan temporel ou logique, ainsi que la raison de certains de ses blocages. La manière, au sens étroit ${ }^{49}$, n'étant qu'une facette d'un même procès (p. ex. parler en élevant la voix), il s'ensuit qu'elle est difficilement exprimable moyennant un tour comme tout + gérondif qui, en revanche, semble instaurer des relations plus indépendantes avec le verbe support.

\section{Références}

AndúJar Moreno, G. 20Io. L'explicitation sémantique dans la traduction français-espagnol des constructions détachées de gérondif. Synergies (Espagne) 3:45-55. En ligne à l'adresse suivante: http://gerflint.fr/Base/Espagnez/moreno.pdf.

Arnavielle, T. 1997. Le morphème -ant, unité et diversité: étude bistorique et théorique. Louvain - Paris : Peeters.

Arnavielle, T. 2003. Le participe, les formes en -ant: positions et propositions. Langages I 49 (I) : 37-54.

Arnavielle, T. 20io. Le gérondif français: nouvelle définition d'un objet étrange. Cabiers AFLS ı6 (I): 6-24. En ligne à l'adresse suivante: http://www.afls.net/ cahiers/ı6.I/3.\%20Arnavielle.pdf.

BARRA-Jover, M. 1995. Quantification et relation interphrastique : à propos du sens oppositif de tout en + gérondif, tout de même, après tout et autres. Travaux de linguistique 30: 5-38.

Bat-Zeev Shyldkrot, H. I995. Tout: polysémie, grammaticalisation et sens prototypique. Langue française 107: 72-92.

BÉGuelin, M.-J. 20I2. Le statut de l'écriture. In R. Druetta (éd.), Claire Blanche-Benveniste: la linguistique à l'école de l'oral. Sylvains-les-Moulins : Éditions Gerflint: 39-54.

Benzitoun, C. 20Io. Comment tirer profit de la parataxe? Étude sur les enchaînements de constructions verbales. In M.-J. Reichler-BÉguelin, M. Avanzi et G. Corminboeuf (éd.), La parataxe. Berne - Berlin - Bruxelles: P. Lang. T. I: Entre dépendance et intégration: $153-174$.

Caron, J. 2008. Précis de psycholinguistique [1989]. Paris: PUF.

Chierchia, G. 1997. Semantica. Bologne: Il Mulino.

Combettes, B. 2003. L'évolution de la forme en -ant: aspects syntaxiques et textuels. Langages I49 (I): 6-24.

47. Pour le français, la bibliographie sur les types de liaison syntaxique est assez riche, surtout grâce au renouvellement apporté par les corpus d'oral spontané (Deulofeu, I999). Pour une mise au point récente, voir Reichler-Béguelin et al. (20I0), dont l'article de Benzitoun (20I0).

48. Comme le démontre Kleiber (2007: 123), le gérondif simple (sans tout) opère sur le mode intégratif et non jonctif. Voir aussi König (1995: 66).

49. Sur les deux façons (étroite et large) d'exprimer la manière moyennant le gérondif simple et sur l'utilisation de tout pour les discriminer, voir Kleiber (201I) et Nádvorníková (2013). 
Condamines, A. 2005. Sémantique et corpus, quelles rencontres possibles? In A. Condamines (éd.), Sémantique et corpus. Paris: Lavoisier - Hermès science publications: 15-38.

Cuniţ̧ă, A. 2orı. C'est en chantant que des muets ont retrouvé l'usage de la parole. Nouveaux regards sur le gérondif. Studii de Lingvistică I : $65-83$. En ligne à l'adresse suivante : http://studiidelingvistica.uoradea.ro/docs/I-20II/pdf_uri/Cunita.pdf.

Deulofeu, H.-J. 1999. Questions de méthode dans la description morphosyntaxique de l'élément que en français contemporain. Recherches sur le français parlé 15: 163-198.

Escoubas-Benveniste, M-P. 20I3. Usages du gérondif et du participe présent en français parlé et écrit: étude comparée basée sur corpus. TIPA - Travaux interdisciplinaires sur la parole et le langage 29. En ligne à l'adresse suivante: http://tipa.revues.org/9o8.

Escoubas-Benveniste, M.-P., Floquet, O. et Bolasco, S. 20i2. Contribution empirique à l'étude du gérondif et du participe présent en français parlé et écrit. In A. Dister, D. LONGRÉE et G. PuRnelle (éd.), Actes des $I^{e}$ journées internationales d'Analyse statistique des données textuelles (JADT 20I2). Liège: Université de Liège: 473-485. En ligne à l'adresse suivante: http://lexicometrica.univ-paris3.fr/jadt/jadt2oI2/Communications/ Escoubas-Benveniste,\%2oMarie-Pierre\%2oet\%2oal.\%20-\%2oContribution\%2o empirique $\% 20 a \%$ 2ol\%27etude\%2odu\%2ogerondif.pdf.

Floquet, O. 20ir. Notes sur la traduction du gerundio. Plaisance 24: 213-226.

Floquet, O., Escoubas-Benveniste, M.-P. et Bolasco, S. 20i2. Sur le gérondif dans le français parlé et écrit. In F. NEveu et al. (éd.), $3^{e}$ congrès mondial de Linguistique française (CMLF 2OI2). Les Ulis: EDP Sciences: 2I43-2154. En ligne à l'adresse suivante : http://www.lettere.uniromar.it/sites/default/files/389/shsconf_cmlfı__ooo247\%20 \%283\%29_o.pdf.

Franckel, J.-J. 1989. Étude de quelques marqueurs aspectuels du français. Genève: Droz.

Fuchs, C. 1982. La paraphrase. Paris : PUF.

Fuchs, C. 1994. Paraphrase et énonciation. Paris - Gap: Ophrys.

Gettrup, H. 1977. Le gérondif, le participe présent et la notion de repère temporel. Revue romane $\mathrm{I} 2(2): 2 \mathrm{II}-270$.

Giacalone Ramat, A. et Mauri, C. 20I2. Gradualness and Pace in Grammaticalization: The Case of Adversative Connectives. Folia Linguistica 46 (2) : 483-512.

Giannini, S. 2003. Il mutamento morfologico. In M. Mancini (éd.), Il cambiamento linguistico. Rome: Carocci : 89-163.

Gleitman, L. R. et Gleitman, H. 1970. Phrase and Paraphrase, some Innovative Uses of Language. New York: W. W. Norton.

Gombert, J.-É. I990. Le développement métalinguistique. Paris: PUF.

Gombert, J.-É. 1996. Activité métalinguistique et acquisition du langage. Acquisition et interaction en langue étrangère $8: 4 \mathrm{I}^{-} 55$. En ligne à l'adresse suivante: http://aile.revues. org/I224.

Halmøy, O. 1990. Réflexions préalables à une nouvelle étude du tour tOUT + GÉRONDIF. Revue romane 25 (2) : I8I-I96.

HaLmøy, O. 2003. Le gérondif en français. Paris - Gap: Ophrys. 
Haspelmath, M. 1995. The Converb as a Cross-Linguistically Valid Category. In E. KöNIG et M. Haspelmath (éd.), Converbs in Cross-Linguistic Perspective: Structure and Meaning of Adverbial Verb Forms - Adverbial Participles, Gerunds. Berlin - New York: Mouton de Gruyter: $\mathbf{1}-55$.

Herslund, M. 2000. Le participe présent comme co-verbe. Langue française I27: 86-94.

KindT, S. 1999. En pleurs vs en pleurant: deux analyses irréconciliables? Travaux de linguistique 38: 109-II8.

KLeIBER, G. 2007. En passant par le gérondif avec mes (gros) sabots. In L. DE SAussure, J. Moeschler et G. PusKás (éd.), Études sémantiques et pragmatiques sur le temps, l'aspect et la modalité. Cahiers Chronos I9. Amsterdam - New York: Rodopi : 93-125.

KLEIBER, G. 20II. Gérondif et manière. Langue française I7I (3) : II7-I34.

KöNIG, E. 1995. The Meaning of Converb Constructions. In E. KöNIG et M. Haspelmath (éd.), Converbs in Cross-Linguistic Perspective: Structure and Meaning of Adverbial Verb Forms - Adverbial Participles, Gerunds. Berlin - New York: Mouton de Gruyter: 57-95.

Le Ny, J.-F. 1987. Sémantique psychologique. In J. A. Rondal et J. P. Thibaut (éd.), Problèmes de psycholinguistique. Bruxelles: P. Mardaga: 13-42.

Lombardi Vallauri, E. 2002. La struttura informativa dell'enunciato. Milan: La Nuova Italia.

Longobardi, G. 2003. La sintassi storica. In M. MANCINI (éd.), Il cambiamento linguistico. Rome: Carocci : $165-208$.

Lonzi, L. 200I. Frasi subordinate al gerundio. In L. Renzi, G. Salvi, A. Cardinaletti (éd.), Grande grammatica italiana di consultazione. Bologne: Il Mulino. Vol. 2: 517-592.

Marchello-Nizia, C. 2007. Le principe de surprise annoncée. Grammaticalisation et pragmaticalisation de «cependant». Discours I. En ligne à l'adresse suivante: http:// discours.revues.org/68.

Mengozzi, A. 1998. Le gérondif et le participe passé dans l'expression du circonstant en italien. In S. Rémi-Giraud et A. Roman (éd.), Autour du circonstant. Lyon: Presses universitaires de Lyon: 28I-293.

MilićEvić, J. 2007. La paraphrase. Berne: P. Lang.

Moeschler, J. 2003. L'expression de la causalité en français. Cabiers de linguistique française 25: II-42. En ligne à l'adresse suivante $:$ http://clf.unige.ch/display.php?numero=25\&idFichier $=56$.

Moline, E. 20II. Peut-on parler de «valeurs sémantiques» du gérondif? In E. ARJACoIEREMIA et al. (éd.), Temps, aspect et classes de mots: études théoriques et didactiques. Arras: Artois Presses Université : 9I-II6.

Morel, M.-A. 1996. La concession en français. Paris - Gap: Ophrys.

Morel, M.-A. et Danon-Boileau, L. 1998. Grammaire de l'intonation. Paris - Gap: Ophrys.

NÁDvorNíKovÁ, O. 2013. "Paul se rase en chantant», dit-il en bafouillant - Quels types de manières pour le gérondif français? Acta Universitatis Carolinae Philologica 2: 3I-44. En ligne à l'adresse suivante: http://www.ceeol.com/aspx/getdocument. aspx?logid $=5 \& \mathrm{zid}=\mathrm{c} 7 \mathrm{ddf} 2 \mathrm{ae}-\mathrm{IcaI}-46$ Iо -8 о Iо- $38 \mathrm{dbb} 97 \mathrm{doc} 6 \mathrm{~d}$.

Nazarenko, A. 2000. La cause et son expression en français. Paris - Gap: Ophrys. 
Nedjalkov, V.P. 1995. Some Typological Parameters of Converbs. In E. KöNig et M. Haspelmath (éd.), Converbs in Cross-Linguistic Perspective: Structure and Meaning of Adverbial Verb Forms - Adverbial Participles, Gerunds. Berlin - New York: Mouton de Gruyter : 97-136.

Oliviéri, M. 20io. Syntaxe et Corpus. Présentation. Corpus 9: 7-20. En ligne à l'adresse suivante: http://corpus.revues.org/ı950.

PInTo, M. A. 1999. La consapevolezza metalinguistica: teoria, sviluppo, strumenti di misurazione. Pise: Istituti editoriali e poligrafici internazionali.

Pinto, M. A. 20ı. Long-Term Effects of Early Bilingualism on Metalinguistic Awareness: A Study on Young Adults. In P. VAlore (éd.), Multilingualism, Language, Power and Knowledge. Pise: Edistudio: 7-27.

Pinto, M. A. et Iliceto, P. 2007. TAM-3 - Test di abilità metalinguistiche n. 3: fascia adolescente-adulta. Rome: Carocci.

Ramat, P. et Da Milano, F. 20ir. Differenti usi di gerundi e forme affini nelle lingue romanze. Vox Romanica 70: I-46.

REICHLER-BÉGuELIN, M.-J. I995. Les problèmes de l'emploi du gérondif et des participiales en français contemporain. In K. ZALESKA et A. CATALDI (éd.), Le français langue étrangère à l'Université: théorie et pratique (Actes du colloque de Varsovie, 25-26 novembre 1993). Varsovie: Uniwersytet Warszawski, Institut Romanistyli : 243-26o.

Reichler-Béguelin, M.-J., Avanzi, M. et Corminboeuf, G. (éd.) 20io. La parataxe. Berne - Berlin - Bruxelles: P. Lang. T. I: Entre dépendance et intégration.

Riegel, M., Pellat, J.-C. et Rioul, R. 2009. Grammaire méthodique du français [1994]. Paris: PUF.

Rins, A. 2009. Gérondif, participe présent et expression de la cause. Nouveaux cabiers de linguistique française 29: 197-2I4. En ligne à l'adresse suivante: http://clf.unige.ch/ display.php?numero=29\&idFichier $=423$.

Rins, A. 20I3. Subjonctif, gérondif et participe présent en français. Une pragmatique de la dépendance verbale. Berne: P. Lang.

SKÅRUP, P. 2006. Les formes déverbales en -ant en ancien français. In C. BARBANCE-GuILlot, S. HeIden et S. Prévost (éd.), À la quête du sens. Études littéraires, bistoriques et linguistiques en hommage à Christiane Marchello-Nizia. Lyon: ENS Éditions: 51-73.

SolARINo, R. 1996. I tempi possibili. Le dimensioni temporali del gerundio italiano. Padoue: Unipress.

Stosic, D. 20I2. En passant par: une expression en voie de grammaticalisation? CORELA - Cognition, représentation, langage HS-I2. En ligne à l'adresse suivante: http://corela.edel.univ-poitiers.fr/index.php?id=2844.

Traugott, E. C. 1989. On the Rise of Epistemic Meanings in English: An Example of Subjectification in Semantic Change. Language 65 (I):3I-55.

VAN VAlin, R. D. Jr. 1984. A Typology of Syntactic Relations in Clause Linkage. In Proceedings of the Ioth Annual Meeting of the Berkeley Linguistics Society. 542-558. En ligne à l'adresse suivante: http://elanguage.net/journals/bls/article/download/2380/2342. 
VIGIER, D. 20I2. En attendant: un cas de pragmaticalisation. Travaux de linguistique 64 (I): I43-I60.

Zanola, M. T. 1998. Gérondif et constructions participiales dans la perspective de l'analyse sémantico-textuelle. In Studi di linguistica francese in Italia 1960-1996 (Actes du colloque de Milan, I7-19 avril 1997). Brescia: La Scuola: 255-266.

Zanola, M. T. 1999. La diachronie des constructions participiales dans une perspective sémantico-textuelle. L'analisi linguistica e letteraria 2 : 333-384.

ZúÑIGA, F. I998. Nomina sunt odiosa: A Critique of the Converb as a Cross-Linguistically Valid Category. Manuscrit. I-I2. En ligne à l'adresse suivante: http://zuniga.unibe.ch/ down/Zuniga-converbs-1998.pdf. 